\title{
Gravitational Collapse of Self-Similar and Shear-free Fluid with Heat Flow
}

\author{
R. Chan ${ }^{1}$, M. F. A. da Silva ${ }^{2}$, and Jaime F. Villas da Rocha ${ }^{2}$ \\ 1 Coordenadoria de Astronomia e Astrofísica, Observatório Nacional \\ Rua General José Cristino 77, São Cristóvão, 20921 - 400 \\ Rio de Janeiro, RJ, Brazil \\ 2 Departamento de Física Teórica, Universidade do Estado do Rio de Janeiro, \\ Rua São Francisco Xavier 524, Maracanã, 20550 - 013 \\ Rio de Janeiro, RJ, Brazil
}

November 2, 2018

\begin{abstract}
A class of solutions to Einstein field equations is studied, which represents gravitational collapse of thick spherical shells made of selfsimilar and shear-free fluid with heat flow. It is shown that such shells satisfy all the energy conditions, and the corresponding collapse always forms naked singularities.
\end{abstract}




\section{Introduction}

One of the most outstanding problems in gravitation theory is the final state of a collapsing massive star, after it has exhausted its nuclear fuel. Despite numerous efforts over the last three decades, our understanding is still limited to several conjectures, such as, the cosmic censorship conjecture [1], and the hoop conjecture [2]. To the former, many counter-examples have been found [3], although it is still not clear whether those particular examples are stable and generic. To the latter, no counter-examples have been found so far in four-dimensional Einstein's Theory of gravity, although it has been shown recently that this is no longer the case in five dimensions [4].

Lately, by studying gravitational collapse of spherically symmetric dust fluid, Joshi, Dadhich and Maartens (JDM) [5] present some results that lead them to the conclusion that the formation of naked singularities is due to shear of the fluid. So, sufficiently strong shearing effects delay the formation of apparent horizons, thereby exposing the strong gravitational regions to the outside world and leading to naked singularities. This is an important and unexpected result, because from the Raychaudhuri equation, one can see that the shear contributes positively to the focusing effect [6]. On the other hand, it is well-known that pressures also play a very significant role in gravitational collapse. Thus, it is natural to ask what kind of roles that shear might play in models with non-vanishing pressures. As pointed out in Ref. [5], when pressures are present the problem becomes very complicated, and it is still not clear which role that shear might play in these models. However, there is at least, as far as we know, two counter-examples to the JDM conjecture $[7,8]$, both of them are particular spherically symmetric models with heat flow (although these authors do not emphasize this relevance of their results). Note also that in both cases their solutions are conformally flat.

In this paper, we shall study a class of solutions, which represents gravitational collapse of a conformally flat, shear-free and self-similar fluid with heat flow. For this class of solutions, we shall show that the collapse always forms naked singularities. Similar results were also found in $[7,8]$. Gravitational collapse of fluid with self-similarity has been intensively studied recently [9]. In addition, gravitational collapse of fluid with heat flow has been also studied lately [10].

The rest of the paper is organized as follows: in Section 2 we shall present the solutions found by Som and Santos (SS) some years ago [11], which 
represents a shear-free fluid with heat flow. We shall show that some of these solutions have self-similarity of the zeroth, first and second kinds, according to the classifications given by Carter and Henriksen [12]. In the study of a fluid with heat flow, one of the difficulties is to verify the energy conditions. Although the problem is simple but often involved very tedious calculations, and sometimes one has to study them numerically [7]. Thus, we devote Section 3 to study the plausible physical conditions $[13,14]$. In the Sections 4 and 5 we present the junction conditions for an exterior Vaidya's spacetime [15] and for an interior Minkowski spacetime. Such constructed solutions can can be considered as representing gravitational collapse of spherical thick shells. It is shown that the collapse always forms naked singularities. The paper is closed by Section 6, in which our main conclusions are presented.

\section{Self-Similar and Shear-free Fluid with Heat Flow}

In this section, we shall study the solutions found by Som and Santos [11], which represent shear-free anisotropic fluid with heat flow. The SS solutions are given by

$$
d s^{2}=A^{2}(t, r)\left(d t^{2}-d r^{2}-r^{2} d \Omega^{2}\right),
$$

where $d \Omega^{2} \equiv d \theta^{2}+\sin ^{2}(\theta) d \phi^{2}, x^{\mu}=\{t, r, \theta, \phi\}$, and

$$
A(t, r)=\frac{1}{f_{1}(t) r^{2}+f_{2}(t)},
$$

with $f_{1}(t)$ and $f_{2}(t)$ being arbitrary functions. This solution has an unusual behavior for the geometrical radius, defined as

$$
\mathbf{R}=\frac{r}{f_{1}(t) r^{2}+f_{2}(t)}
$$

As a matter of fact, when $r \rightarrow 0$ we have $\mathbf{R} \rightarrow 0$. In addition, if $f_{1}(t) f_{2}(t)>0$, we have $\mathbf{R} \rightarrow 0$, too, as $r \rightarrow \infty$. Then, at any given moment $t=t_{1}$, we can see that $\mathbf{R}$ is always bounded, as can be seen from the figure (1).

The corresponding energy-momentum tensor (EMT) takes the form ${ }^{1}$,

$$
T_{\mu \nu}=\rho u_{\mu} u_{\nu}-\tilde{p} h_{\mu \nu}+q_{\mu} u_{\nu}+q_{\mu} u_{\nu}
$$

\footnotetext{
${ }^{1}$ In this paper we shall choose units such that the Einstein coupling constant $\kappa$ is unity.
} 


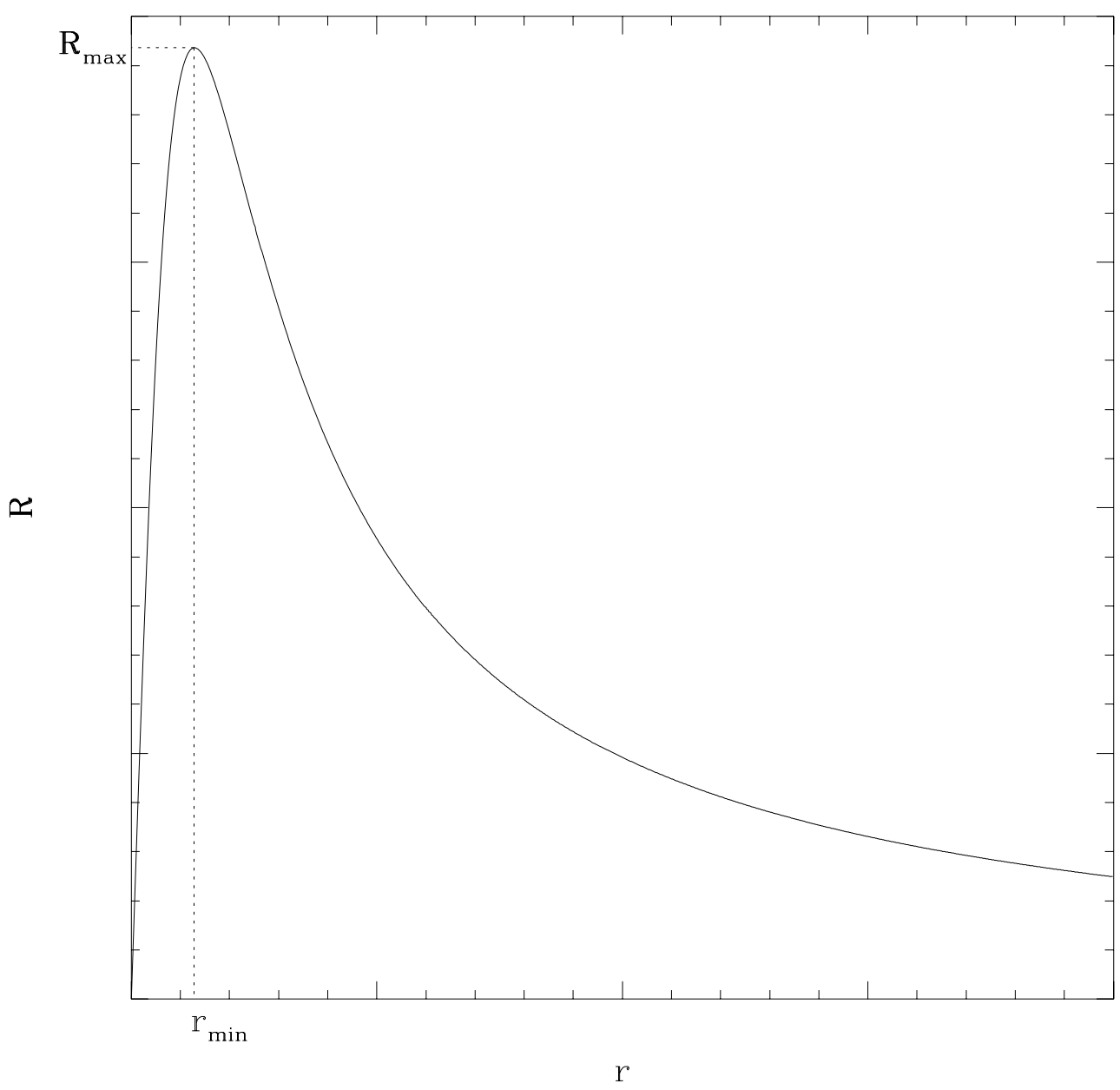

Figure 1: The geometrical radius $\mathbf{R}$ as a function of the coordinate $r$. For $r \leq r_{\text {min }}$, we have $r=\left(1-\sqrt{1-4 f_{1} f_{2} \mathbf{R}^{2}}\right)\left(2 f_{1} \mathbf{R}^{2}\right)^{-1}$ and $r \geq r_{\text {min }}$, we have $r=\left(1+\sqrt{1-4 f_{1} f_{2} \mathbf{R}^{2}}\right)\left(2 f_{1} \mathbf{R}^{2}\right)^{-1}$ where $r_{\text {min }}=\sqrt{f_{2} / f_{1}}$. The maximal geometrical radius, $\mathbf{R}_{\max }=\mathbf{R}\left(r_{\min }\right)$, is given by $(-t) /(2 \sqrt{c})$. 
where $u_{\nu}$ is the four-velocity of the fluid, $\rho$ the energy density measured by comoving observers with the fluid, $h_{\mu \nu}$ denotes the inducted metric on the three-surfaces orthogonal to $u_{\nu}$, and $\tilde{p}$ is the effective pressure. In terms of the isotropic pressure $p$ and the second coefficient of the viscosity, $\zeta, \tilde{p}$ is given by $\tilde{p}=p-\zeta u_{; \mu}^{\mu}$. In equation (4) the heat flow vector $q^{\mu}$ satisfies the condition $q^{\mu} u_{\mu}=0$ and in the comoving coordinates is given by

$$
q^{\mu}=-\frac{4 r}{k A^{2}} f_{1}^{\prime}(t) \delta_{r}^{\mu}
$$

where a prime denotes the ordinary differentiation with respect to $t$. For the details, we refer readers to Ref. [11].

In this paper, we shall consider a particular case of the SS solutions (2), where the fluid is not viscous and the corresponding spacetime has selfsimilarity.

Generalizing the concepts of Newtonian Mechanics [16] to General Relativity, Carter and Henriksen $(\mathrm{CH})[12]$ gave the notion of kinematic selfsimilarity with its properties,

$$
\mathcal{L}_{\xi} h_{\mu \nu}=2 h_{\mu \nu}, \quad \mathcal{L}_{\xi} u^{\mu}=-\alpha u^{\mu}
$$

where $\mathcal{L}_{\xi}$ is a Lie operator, $h_{\mu \nu}$ is the project operator, defined by $h_{\mu \nu}=g_{\mu \nu}$ - $u_{\mu} u_{\nu}$, and $\alpha$ is an arbitrary constant. When $\alpha=1$, it can be shown that the kinematic self-similarity reduces to the homothetic one (or self-similarity of the first kind), which was first studied by Cahill and Taub in GR for a perfect fluid [17]. When $\alpha \neq 1, \mathrm{CH}$ argued that this would be a natural relativistic counterpart of self-similarity of the second kind $(\alpha \neq 1)$ and of the zeroth kind $(\alpha=0)$ in Newtonian Mechanics.

Applying the conditions (6) to the metric (1), it can be shown that the metric coefficient $A(t, r)$ has to take the form,

$$
A(t, r)=A(z)
$$

where in each case the conform Killing vector $\xi^{\mu}$ and the self-similar variable $z$ are given by

$$
\xi^{\mu} \frac{\partial}{\partial x^{\mu}}=\frac{\partial}{\partial t}+r \frac{\partial}{\partial r}, \quad z=r e^{-t},(\alpha=0)
$$


for the zeroth kind, and

$$
\xi^{\mu} \frac{\partial}{\partial x^{\mu}}=\alpha t \frac{\partial}{\partial t}+r \frac{\partial}{\partial r}, \quad z=\frac{r}{(-t)^{1 / \alpha}},(\alpha \neq 0),
$$

for the second kind. In the latter case when $\alpha=1$ it degenerates to the first kind. For the details, we refer to $[12,18,19]$.

\subsection{Solutions with Self-similarity of the Zeroth Kind}

¿From Eqs. (8) and (7), we can see that for the SS solutions (2), the ones with self-similarity of the zeroth kind are given by

$$
f_{1}(t)=e^{-2 t}, \quad f_{2}(t)=c,
$$

where $c$ is an arbitrary constant. The spacetime singularities are manifested from the associated Kretschmann scalar,

$$
\begin{aligned}
\mathcal{K}= & R^{\mu \nu \lambda \sigma} R_{\mu \nu \lambda \sigma}=-16 e^{-8 t}\left\{\left(12 r^{4}-28 r^{2}+15\right) c^{2} e^{-4 t}\right. \\
& \left.+r^{2}\left[c e^{2 t}\left(4 r^{2}-6\right)+r^{2}\left(12 r^{4}-16 r^{2}+3\right)\right]\right\},
\end{aligned}
$$

from which we can see that it is singular at $t=-\infty$ and $r=\infty(\mathbf{R}=0)$. It can be shown that in this case the solutions cannot be interpreted as representing gravitational collapse. Thus, in the following we shall not consider them any more.

\subsection{Solutions with Self-similarity of the First and Sec- ond Kinds}

The SS solutions with self-similarities of the first and second kinds are given by

$$
f_{1}(t)=(-t)^{-2 / \alpha} \quad f_{2}(t)=c,
$$

where $c$, as in the last case, is a constant. Then, it can be shown that the corresponding Kretschmann scalar reads

$$
\mathcal{K}=-\frac{16}{\alpha^{4}(-t)^{4(2+\alpha) / \alpha}}\left\{c ^ { 2 } ( - t ) ^ { 4 / \alpha } \left[15(\alpha t)^{4}\right.\right.
$$




$$
\begin{aligned}
& \left.-2 \alpha^{3}(3 \alpha+14) t^{2} r^{2}+3\left(\alpha^{2}+2\right)^{2} r^{4}\right] \\
& +c r^{2}(-t)^{2 / \alpha}\left[6 \alpha^{2} r^{4}-6(\alpha t)^{4}+4 t^{2} r^{2}+12 \alpha r^{4}\right] \\
& \left.+r^{4}\left[3(\alpha t)^{4}+2 \alpha^{2}(3 \alpha-8) r^{2} t^{2}+3\left(\alpha^{2}+4\right) r^{4}\right]\right\}
\end{aligned}
$$

which shows that the spacetime now is singular at $t=0$.

The apparent horizon of the fluid can be defined as the outer hypersurface that satisfies the equation [20]

$$
\mathbf{R}_{, \alpha} \mathbf{R}_{, \beta} g^{\alpha \beta}=0 .
$$

It will be shown below that the apparent horizon of the fluid is always outside of the three energy condition frontiers. Besides, from equation (3), we can see that the geometrical radius vanishes when $t=0$. Figures (2), (3) and (4) show the solutions of equation (14) for various values of $c$ and $\alpha$. Their comparison, and the fact that the case with $\alpha=1$ (first kind of self-similarity) can be considered as a sub-case of the second kind lead to the conclusion that the cases with first and second kind of self-similarities present the same physics. So, in the following, we will study only the solutions with first kind.

It should be noted that to consider these solutions as physical, they have to satisfy some physical and geometrical conditions $[13,14]$. We shall devote the next section to study these conditions. Before proceeding, we would also like to note that the spacetimes of the above solutions are singular at $z=\infty$, too, as one can see from Eqs.(11) and (13). However, as we shall show below, the energy conditions will restrict these solutions valid only in the region $z_{1}<z<z_{2}$, where $z_{1,2}$ are finite constants. Thus, the singularity at $z=\infty$ becomes irrelevant in the models to be constructed below. 


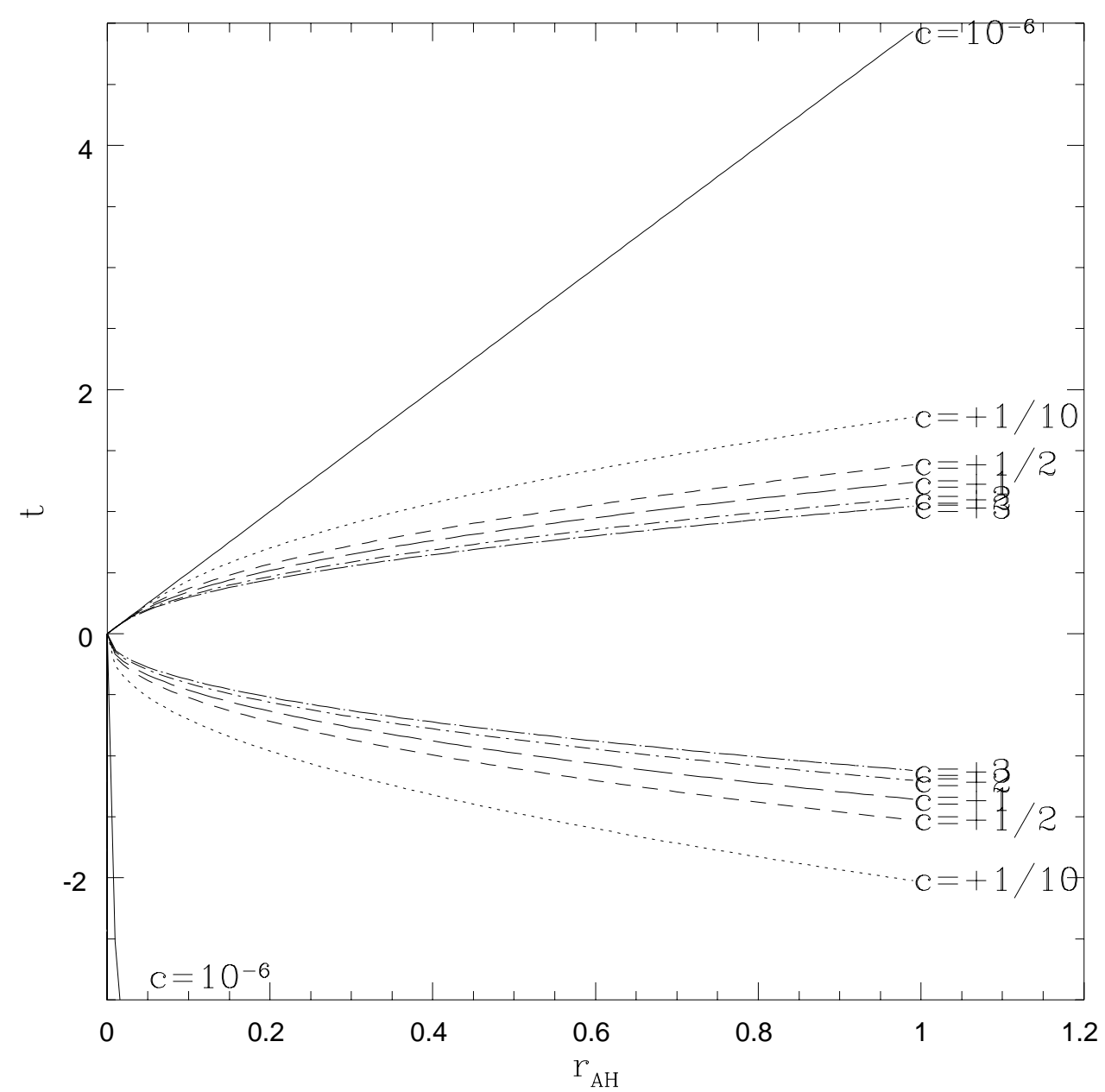

Figure 2: The apparent horizon radius for $\alpha=2 / 5$. 


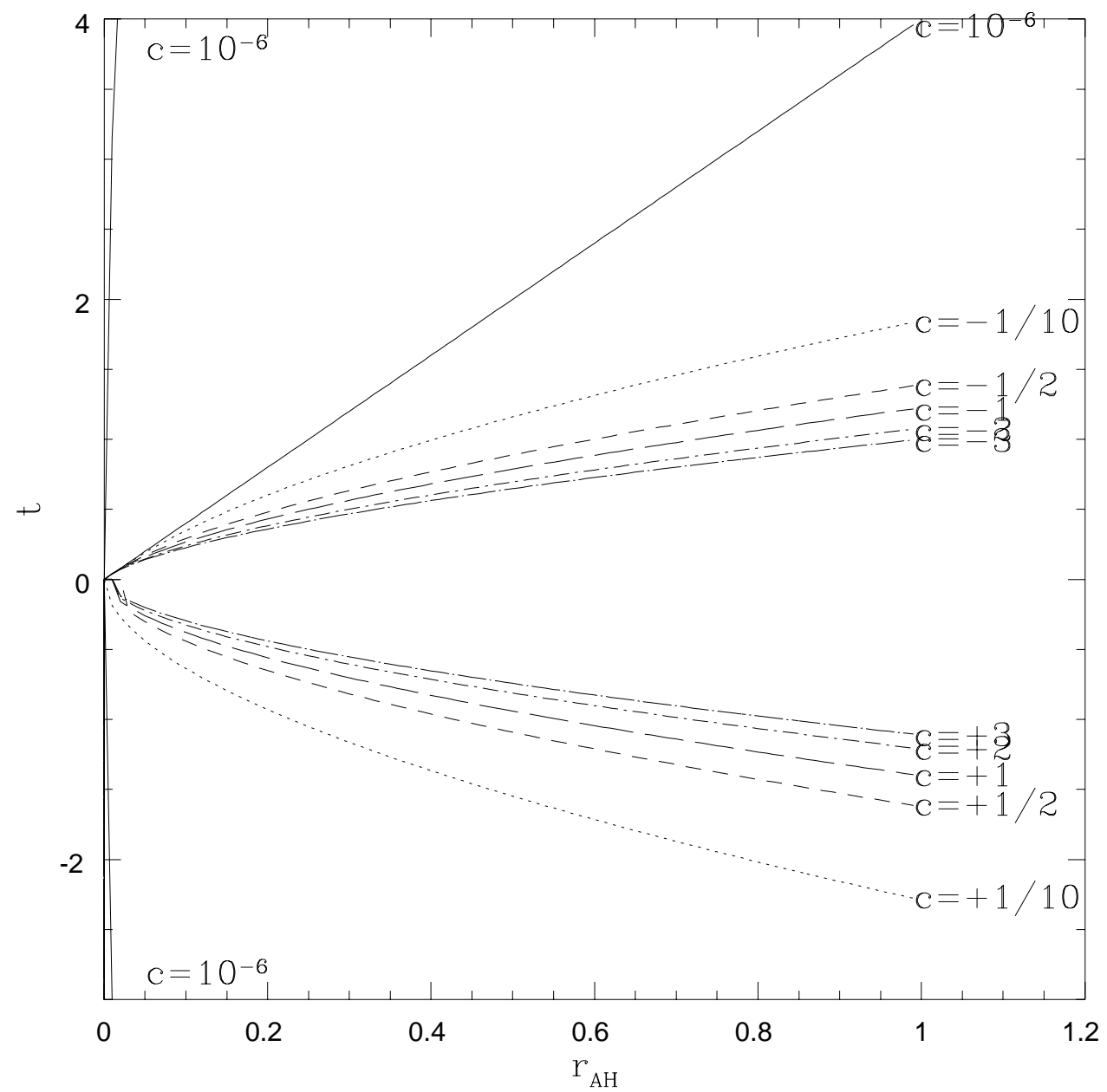

Figure 3: The apparent horizon radius for $\alpha=1 / 2$. 


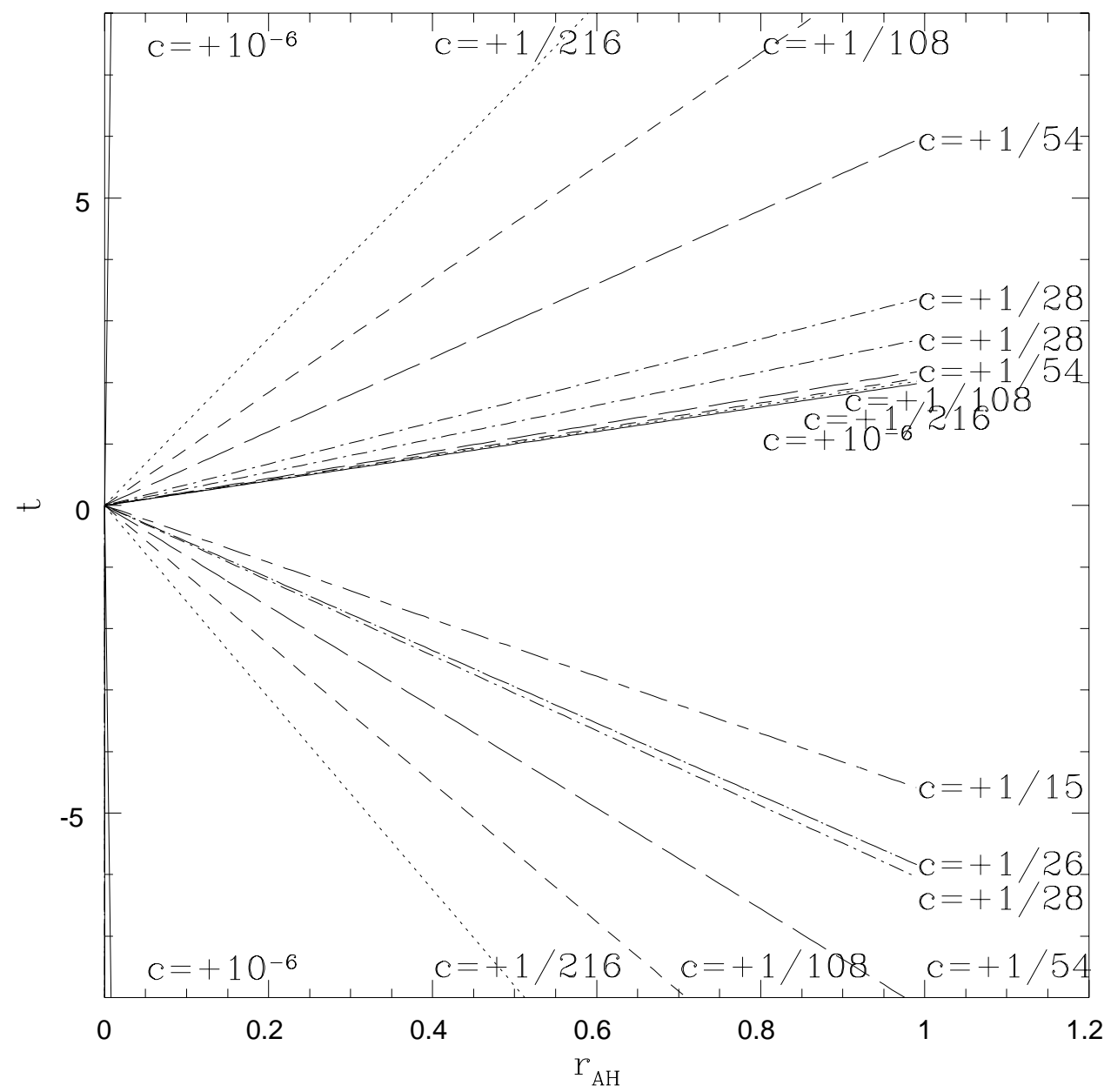

Figure 4: The apparent horizon radius for $\alpha=1$. 


\section{Plausible Physical Conditions}

Although the solution (2), at first sight, is mathematically very simple, in virtue of the difficulties imposed by (3), we need to enterprise a very careful analysis, in order to assure their correct interpretation and, with this, select those subsets of solutions that can represent astrophysical collapse.

\subsection{Energy Conditions}

Since the corresponding EMT has heat flow and, as a result, it is not diagonal, then the analysis of the energy conditions becomes considerably complicated. In order to study these energy conditions, one has first to cost it in its canonical form [13]. We find that solutions with self-similarity of the first kind have similar properties as those with self-similarity of the second and zeroth kinds. Thus, in the following we shall consider only the solutions with $\alpha=1$. For these solutions it can be shown that the EMT given by equation (4) can be written as,

$$
T_{\mu \nu}=\rho t_{\mu} t_{\nu}+q\left(t_{\mu} r_{\nu}+r_{\mu} t_{\nu}\right)+p\left(\theta_{\mu} \theta_{\nu}+\phi_{\mu} \phi_{\nu}\right),
$$

where $t_{\mu}=A \delta_{\mu}^{t}, r_{\mu}=A \delta_{\mu}^{r}, \theta_{\mu}=r A \delta_{\mu}^{\theta}, \phi_{\mu}=r A \sin \theta \delta_{\mu}^{\phi}$, and

$$
\begin{aligned}
\rho & =\frac{12}{t^{2}}\left(c+z^{4}\right), \quad q=-\frac{8 z}{t^{2}}\left(c+z^{2}\right)^{2}, \\
p & =\frac{4}{t^{2}}\left[-2 c+(1+3 c) z^{2}\right]
\end{aligned}
$$

where $z=r /(-t)$, as defined in equation (9), is the self-similar variable. To write the EMT in its canonical form, we need to solve the eigenvalue problem,

$$
\tau_{\nu}^{\mu} \xi^{\nu}=\lambda \xi^{\mu}
$$

which will possess nontrivial solutions only when the determinant $\operatorname{det} \mid \tau_{\nu}^{\mu}-$ $\lambda \delta_{\nu}^{\mu} \mid=0$ that can be written as [21],

$$
(\lambda+p)^{2}\left[(\lambda-\rho)(\lambda+p)+q^{2}\right]=0 .
$$

Clearly, the above equation has four roots, $\lambda_{1,2}=p$ and $\lambda_{ \pm}$, where

$$
\lambda_{ \pm}=\frac{(\rho-p)}{2} \pm \Delta, \quad \Delta^{2} \equiv \frac{(\rho+p)^{2}-4 q^{2}}{4} .
$$


The eigenvalues $\lambda_{1,2}$ correspond to, respectively, the eigenvector $\xi_{2}^{\mu}=\theta^{\mu}$ and $\xi_{3}^{\mu}=\phi^{\mu}$, which represent the two principal tangent directions. On the other hand, substituting equation (19) into equation (17), we find that the corresponding eigenvectors are given by

$$
\xi_{ \pm}^{\mu}=\left(\lambda_{ \pm}+p\right) t^{\mu}+q r^{\mu}
$$

The conditions $\Delta^{2}>0, \Delta^{2}=0$ and $\Delta^{2}<0$ divide the whole spacetime into several regions. In the following let us first consider regions where $\Delta^{2}>$ 0 .

\subsubsection{Regions with $\Delta^{2}>0$}

¿From equation (19) we find that

$$
\Delta^{2}=\frac{4}{t^{4}}\left(9 z^{2}-1\right)\left(z^{2}-1\right)\left(z^{2}+c\right)^{2} .
$$

Thus, the regions where $\Delta^{2}>0$ depend on the values of the constant $c$. In particular, we find that

$$
=> \begin{cases}z<1 / 3, \text { or } z>1, & c>0, \\ z<\sqrt{-c} \text { or } z>1 \text { or } \sqrt{-c}<z<1 / 3 \text { or } 1 / 3<z<1, & -1 / 9<c<0, \\ z<1 / 9 \text { or } z>1 \text { or } 1 / 3<z<\sqrt{-c} \text { or } \sqrt{-c}<z<1, & -1<c<-1 / 9, \\ z<1 / 9 \text { or } z>\sqrt{-c} \text { or } 1 / 3<z<1 \text { or } 1<z<\sqrt{-c} & c<-1 .\end{cases}
$$

As can be seen from equation (19), now the two roots $\lambda_{ \pm}$and the two eigenvectors $\xi_{ \pm}^{\mu}$ are all real and satisfy the relations,

$$
\begin{aligned}
\left(\lambda_{+}+p\right)\left(\lambda_{-}+p\right) & =q^{2}, \\
\frac{\xi_{ \pm}^{\mu} \xi_{ \pm}^{\nu} g_{\mu \nu}}{\Delta\left(\lambda_{ \pm}+p\right)} & = \pm 1, \\
\xi_{+}^{\mu} \xi_{-}^{\nu} g_{\mu \nu} & =0 .
\end{aligned}
$$

¿From these expressions we can see that when $\lambda_{+}+p>0$, the eigenvector $\xi_{+}^{\mu}$ is timelike, and $\xi_{-}^{\mu}$ is spacelike, while when $\lambda_{+}+p<0$, the two vectors exchange their roles.

Case A.1) $\lambda_{+}+p>0$ : This condition can be written as

$$
\rho+p+2 \Delta>0
$$


Setting

$$
\begin{aligned}
E_{(0)}^{\mu} & \equiv \frac{\xi_{+}^{\mu}}{\left[D^{1 / 2}\left(\lambda_{+}+p\right)\right]^{1 / 2}}, \\
E_{(1)}^{\mu} & \equiv \frac{\xi_{-}^{\mu}}{\left[D^{1 / 2}\left(\lambda_{-}+p\right)\right]^{1 / 2}}, \\
E_{(2)}^{\mu} & \equiv \theta^{\mu}, E_{(3)}^{\mu} \equiv \phi^{\mu}
\end{aligned}
$$

we find that $E_{(a)}^{\mu},(a=0,1,2,3)$ form an orthogonal basis, i.e., $E_{(a)}^{\lambda} E_{(b) \lambda}=$ $\eta_{a b}$, with $\eta_{a b}=$ diag. $\{1,-1,-1,-1\}$. Then, in terms of these unit vectors, the EMT given by equation (15) takes the form

$$
\left(T_{(a)(b)}\right)=\left(\begin{array}{cccc}
\rho_{(0)} & 0 & 0 & 0 \\
0 & p_{(1)} & 0 & 0 \\
0 & 0 & p_{(2)} & 0 \\
0 & 0 & 0 & p_{(3)}
\end{array}\right),
$$

where $T_{(a)(b)} \equiv T_{\mu \nu} E_{(a)}^{\mu} E_{(b)}^{\nu}$, and

$$
\begin{aligned}
& \rho_{(0)}=\frac{1}{2}(\rho-p+2 \Delta), \\
& p_{(1)}=\frac{1}{2}(p-\rho+2 \Delta), \\
& p_{(2)}=p_{(3)}=p .
\end{aligned}
$$

equation (26) corresponds to the Type I fluid defined in [13].

Once the EMT is cast in its canonical form, we can apply the energy conditions to it. A simple but tedious analysis of these conditions reveals that all of them are satisfied only for the case where $0<c<1 / 15$ and $z_{1}<z<z_{2}$ as showed in Fig. 5. The hypersurfaces $z=z_{1,2}$ are determined as follows:

The boundary $z=z_{1}$ comes from the condition $\rho_{(0)}+p_{(1)}+2 p \geq 0$, and is given by the first positive root of the equation

$$
9 x^{4}+2(9 c-5) x^{3}-\left(3+44 c+27 c^{2}\right) x^{2}+(18+38 c) c x-15 c^{2}=0,
$$

while the boundary $z=z_{2}$ is obtained from the condition $\rho_{(0)}-p \geq 0$ and given by the second positive root of the equation

$$
(1+9 c) x^{3}-\left[(1+3 c)^{2}+10 c\right] x^{2}+c[7(1+3 c)+2 c] x-10 c^{2}=0,
$$




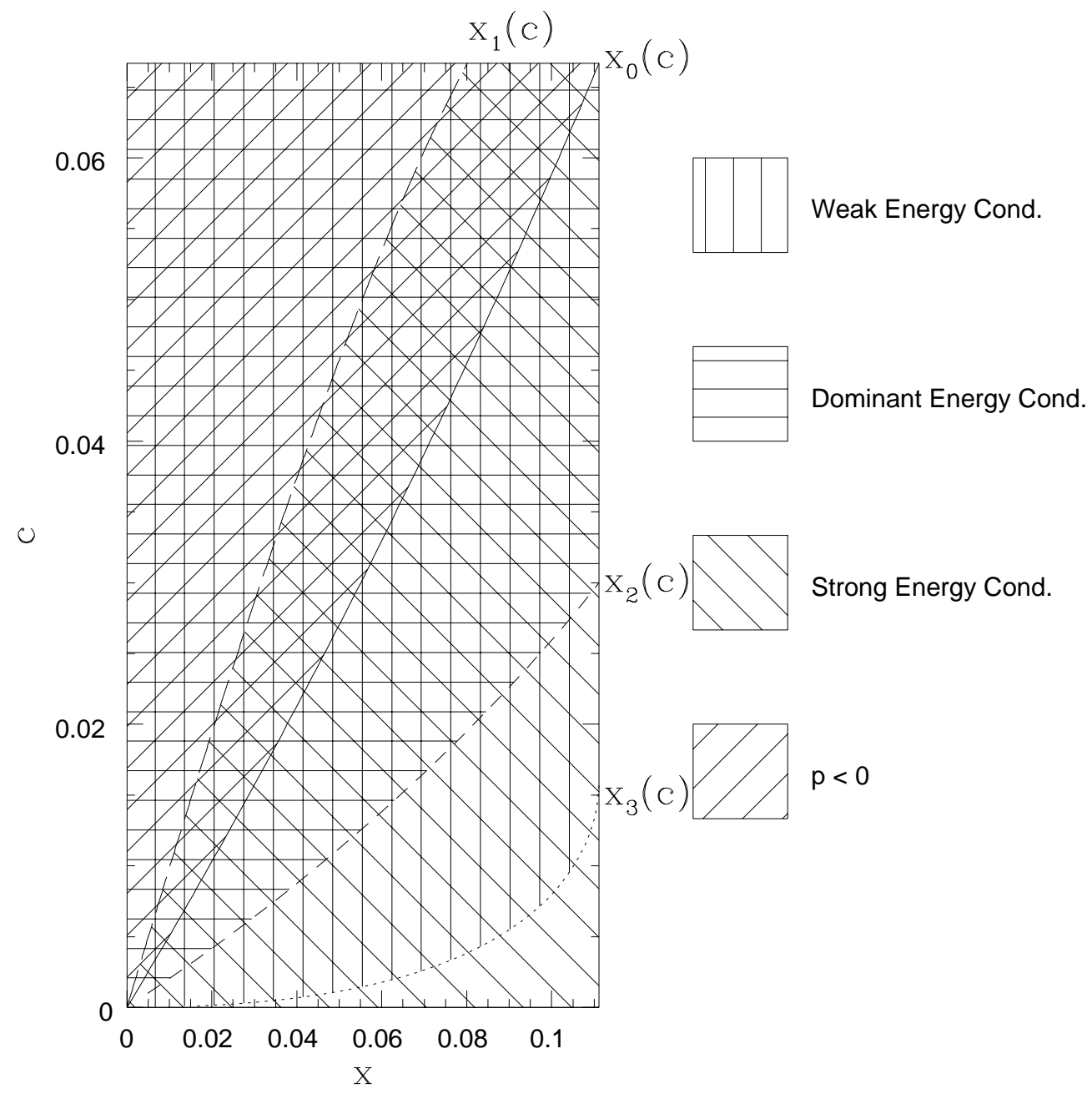

Figure 5: The different intervals where the energy conditions are satisfied. For the range $\mathrm{x}$ between $\mathrm{x}_{1}$ and $\mathrm{x}_{2}$ where $\mathrm{x}=z^{2}$ all the three energy conditions are satisfied 
where $x \equiv z^{2}$. In between these two boundaries, all the rest of energy conditions are identically satisfied.

Case A.2) $\lambda_{+}+p<0$ : This condition can be written as

$$
\rho+p+2 \Delta<0
$$

Since now $\xi_{-}^{\mu}$ is time-like, the orthogonal basis can be chosen as

$$
E_{(a)}^{\mu} \equiv\left\{\frac{\xi_{-}^{\mu}}{D^{1 / 4}\left|\lambda_{-}+p\right|^{1 / 2}}, \frac{\xi_{+}^{\mu}}{D^{1 / 4}\left|\lambda_{+}+p\right|^{1 / 2}}, \theta^{\mu}, \phi^{\mu}\right\} .
$$

Then, it can be shown that the corresponding EMT also takes the form of equation (26) but now with

$$
\begin{aligned}
& \rho_{(0)}=\frac{1}{2}(\rho-p-2 \Delta), \\
& p_{(1)}=\frac{1}{2}(p-\rho-2 \Delta), \\
& p_{(2)}=p_{(3)}=p .
\end{aligned}
$$

¿From equation (30) one can show that the weak and dominant energy conditions are violated for any given values of $c$. Thus, in the following discussions we shall discard this case.

Summarizing the results of this subsection we can see that the fluid satisfies all the three energy conditions only in the region where $z_{1}<z<z_{2}$ or equivalently, $r_{1}(t)<r<r_{2}(t)$, where $r_{1}(t)=-z_{1} t$ and $r_{2}(t)=-z_{2} t$.

\subsubsection{The Hypersurfaces where $\Delta^{2}=0$}

¿From equation $(21)$ we can see that now $\Delta^{2}=0$ represents the hypersurfaces ${ }^{2}$ $z=1 / 3,1, \sqrt{-c}$, on which we have

$$
q=-\frac{1}{2}(\rho+p)
$$

and the two roots $\lambda_{ \pm}$given by equation (19) degenerate into one. As shown in [21], this multiple root corresponds to two null independent eigenvectors,

$$
\xi_{ \pm}^{\mu}=\frac{u^{\mu} \pm X^{\mu}}{\sqrt{2}} .
$$

\footnotetext{
${ }^{2}$ Since in this paper we are mainly concerned with gravitational collapse of the fluid, we consider only the region where $t \leq 0$ or $z \geq 0$.
} 
¿From these two null vectors we can construct two unit vectors, one is timelike and the other is spacelike, but these are exactly $u^{\mu}$ and $r^{\mu}$. Thus, in the basis

$$
E_{(a)}^{\mu}=\left\{u^{\mu}, r^{\mu}, \theta^{\mu}, \phi^{\mu}\right\},
$$

the EMT takes the form

$$
\left(T_{(a)(b)}\right)=\left(\begin{array}{cccc}
\rho & q & 0 & 0 \\
q & p & 0 & 0 \\
0 & 0 & p & 0 \\
0 & 0 & 0 & p
\end{array}\right)
$$

To consider the energy conditions on these hypersurfaces, it is found convenient to distinguish the three cases $q>0, q=0$ and $q<0$.

Case B.1) $q>0$ : In this case, the corresponding EMT (36) can be written in the form

$$
\left(T_{(a)(b)}\right)=q\left(\begin{array}{cccc}
1+\kappa & 1 & 0 & 0 \\
1 & 1-\kappa & 0 & 0 \\
0 & 0 & p_{(2)} & 0 \\
0 & 0 & 0 & p_{(3)}
\end{array}\right), \quad(q>0),
$$

where

$$
\kappa \equiv \frac{\rho-p}{\rho+p}, \quad p_{(2)}=p_{(3)} \equiv \frac{2 p}{\rho+p} .
$$

equation (37) is exactly in the form of the type II fluid classified in [13]. Applying the three energy conditions to this case we find that all of them are satisfied for $z=1, c<-1$ and $z=1 / 3, c<1 / 63$.

Case B.2) $q=0$ : In this case it can be shown that the EMT becomes diagonal and the energy conditions are these given for the EMT of equation (26). Then, it can be shown that only on the hypersurface $z=1$ with $c=-1$ all the three energy conditions are satisfied.

Case B.3) $q<0$ : In this case, it can be shown that the corresponding EMT cannot be written in the form of equation (37). In order to study the energy conditions, let us consider an observer with its four-velocity given by

$$
w^{\mu}=\alpha t^{\mu}+\beta r^{\mu}+\gamma \theta^{\mu}+\delta \phi^{\mu},
$$

where $\alpha, \beta, \gamma$ and $\delta$ are arbitrary constants, subject to the condition,

$$
w^{\mu} w_{\mu}=\alpha^{2}-\beta^{2}-\gamma^{2}-\delta^{2} \geq 0 .
$$


The weak energy condition requires that [13]

$$
T_{\mu \nu} w^{\mu} w^{\nu}=\alpha^{2} \rho+\gamma^{2} p+\delta^{2} p-2 \alpha \beta q \geq 0 .
$$

It can be shown that equation (41) is satisfied for any observer given by Eqs.(39) and (40) only when the conditions $\rho \geq 0, \rho+p \geq 0, \rho+p-2 q \geq 0$ and $\rho+p+2 q \geq 0$ are true. On the other hand, the strong energy condition holds when [13]

$$
\begin{aligned}
\left(T_{\mu \nu}-\frac{1}{2} g_{\mu \nu} T\right) w^{\mu} w^{\nu}= & \frac{1}{2}\left[\left(\alpha^{2}+\beta^{2}+\gamma^{2}+\delta^{2}\right) \rho\right. \\
& +\left(\alpha^{2}+\gamma^{2}-\beta^{2}-\delta^{2}\right) p \\
& \left.+\left(\alpha^{2}+\delta^{2}-\beta^{2}-\gamma^{2}\right) p-4 \alpha \beta q\right] \geq 0,
\end{aligned}
$$

which is equivalent to $\rho+p \geq 0, \rho+p-2 q \geq 0, \rho+p+2 q \geq 0$ and $\rho+p+p \geq 0$. Meanwhile, the dominant energy condition requires that $\rho \geq|p|, \rho \geq|p|$ and $\rho \geq|q|$. To summarize, for any given $T_{\mu \nu}$ of the form (36), the energy conditions are the following:

(a) The Weak Energy Condition:

$$
\text { i) } \rho \geq 0, \text { ii) } \rho+p \geq 0, \text { iii) } \rho+p+2 q \geq 0, \text { iv) } \rho+p-2 q \geq 0 \text {. }
$$

(b) The Dominant Energy Condition:

$$
\text { i) } \rho \geq|p|, \quad \text { ii) } \rho \geq|p|, \quad \text { iii) } \rho \geq|q| \text {. }
$$

(c) The Strong Energy Condition:

$$
\begin{aligned}
& \text { i) } \rho \geq 0, \quad \text { ii) } \rho+p \geq 0, \quad \text { iii) } \rho+p-2 q \geq 0 \\
& \text { iv) } \rho+p+2 q \geq 0, \quad v) \rho+2 p \geq 0 .
\end{aligned}
$$

Applying the above energy conditions to the fluid, we find that it satisfies all of them only on the hypersurfaces $z=1$ with $c \geq-1$ and $z=1 / 3$ with $1 / 18 \leq c \leq 7 / 9$.

In summary, in the present case we have only isolated hypersurfaces satisfying all the energy conditions. 


\subsubsection{Regions with $\Delta^{2}<0$}

¿From Eqs.(19) and (20), we can see that now the eigenvalues $\lambda_{ \pm}$are complex, and so do the two eigenvectors $\xi_{ \pm}^{\mu}$. This means that in the present case the EMT cannot be diagonalized (by real similarity transformations). Then, in terms of the four unit vectors $\left\{u^{\mu}, r^{\mu}, \theta^{\mu}, \phi^{\mu}\right\}$, it will take exactly the same form as that given by equation (36) but now with $q$ being arbitrary. The analysis of the three energy conditions will follow precisely the same steps as we did in the last subcase for $q<0$, so the three energy conditions are also those given by Eqs.(43)-(45).

It is not difficult to show that in this case none of these three energy conditions is satisfied. In fact, from the condition $\Delta^{2}<0$ we find that

$$
(\rho+p)^{2}-4 q^{2}<0
$$

while the weak energy and strong conditions all require $(\rho+p)^{2}-4 q^{2} \geq 0$. Since the dominant energy condition is stronger than the weak one, we can see that in the present case, all the three energy conditions are violated.

Thus, from the above analysis we can see that, in order to interpret the self-similar fluid given by Eqs.(10) and (12) as representing gravitational collapse, the solutions need to be restricted to certain regions. For the homothetic case, we have shown that it is given by $z_{1}<z<z_{2}$ and $-\infty<t \leq 0$, where $z_{1}<z_{2}<1 / 3$. Outside of this regions, the spacetime will be described by other solutions. For example, if we consider the hypersurface $z=z_{1}$, or $r=r_{1}(t)$ then we may match it to a Schwarzschild, Vaidya, or any region of other spacetimes for $r<r_{1}(t)$ or $\mathbf{R}>\mathbf{R}_{1}(t)$. On the other hand, inside of the fluid $r>r_{2}(t)$ or $\mathbf{R}<\mathbf{R}_{2}(t)$, the spacetime can be matched to Minkowski spacetime. Since the hypersurfaces $z=z_{1,2}$ or $r=r_{1,2}(t)$ are time-like, in principle these matchings are always possible. Then, the resultant spacetime will represent the collapse of a shell of a fluid with finite thickness. The spacetime is free of singularities at initial, but due to the collapse of the fluid, a spacetime singularity will be finally formed at the moment $t=0$ at the origin $\mathbf{R}=0$. This singularity is naked and it is never covered by a horizon as it will be shown below. 


\section{2 "Astrophysical" Conditions}

Besides the energy conditions, we consider three additional physical plausible conditions [14] which allow us to associate the source studied here with a stellar object. These conditions are

$$
\frac{\partial \rho}{\partial \mathbf{R}}<0, \quad p>0, \quad \frac{\partial p}{\partial \mathbf{R}}<0, \quad \frac{\partial\left(q_{\alpha} q^{\alpha}\right)}{\partial \mathbf{R}}>0 .
$$

The graphics in the figure (6) show clearly that these conditions are fulfilled.

\section{Matching Regions I and III}

In this section we will present the junction conditions for the regions I (Minkowski spacetime) and III (fluid with heat flow) and for the regions III (fluid with heat flow) and V (Vaidya's spacetime), as shown in the figure 7 .

\subsection{Junction Conditions for the region II}

The Minkowski metric can be written as

$$
d s_{I}^{2}=d V^{2}-d R^{2}-R^{2} d \theta^{2}-R^{2} \sin ^{2} \theta d \phi^{2},
$$

and the shell fluid can be written as

$$
d s_{I I I}^{2}=A^{2}(r, t)\left[d t^{2}-d r^{2}-r^{2} d \theta^{2}-r^{2} \sin ^{2} \theta d \phi^{2}\right] .
$$

The metric of the junction hypersurface $\Sigma_{1}$ is given by

$$
d s_{I I}^{2}=d \tau^{2}-\beta^{2}(\tau)\left(d \theta^{2}+\sin ^{2} \theta d \phi^{2}\right),
$$

where the time coordinate $\tau$ is defined only on $\Sigma_{1}$.

Thus, using the first fundamental forms on $\Sigma_{1}$ we have

$$
\left(d s_{I}^{2}\right)_{\Sigma_{1}}=\left(d s_{I I}^{2}\right)_{\Sigma_{1}}=\left(d s_{I I I}^{2}\right)_{\Sigma_{1}}
$$

resulting

$$
\left[1-\left(\frac{d R}{d V}\right)_{\Sigma_{1}}^{2}\right] d V^{2}=d \tau^{2}=A^{2}\left(r_{\Sigma_{1}}, t\right) d t^{2}
$$




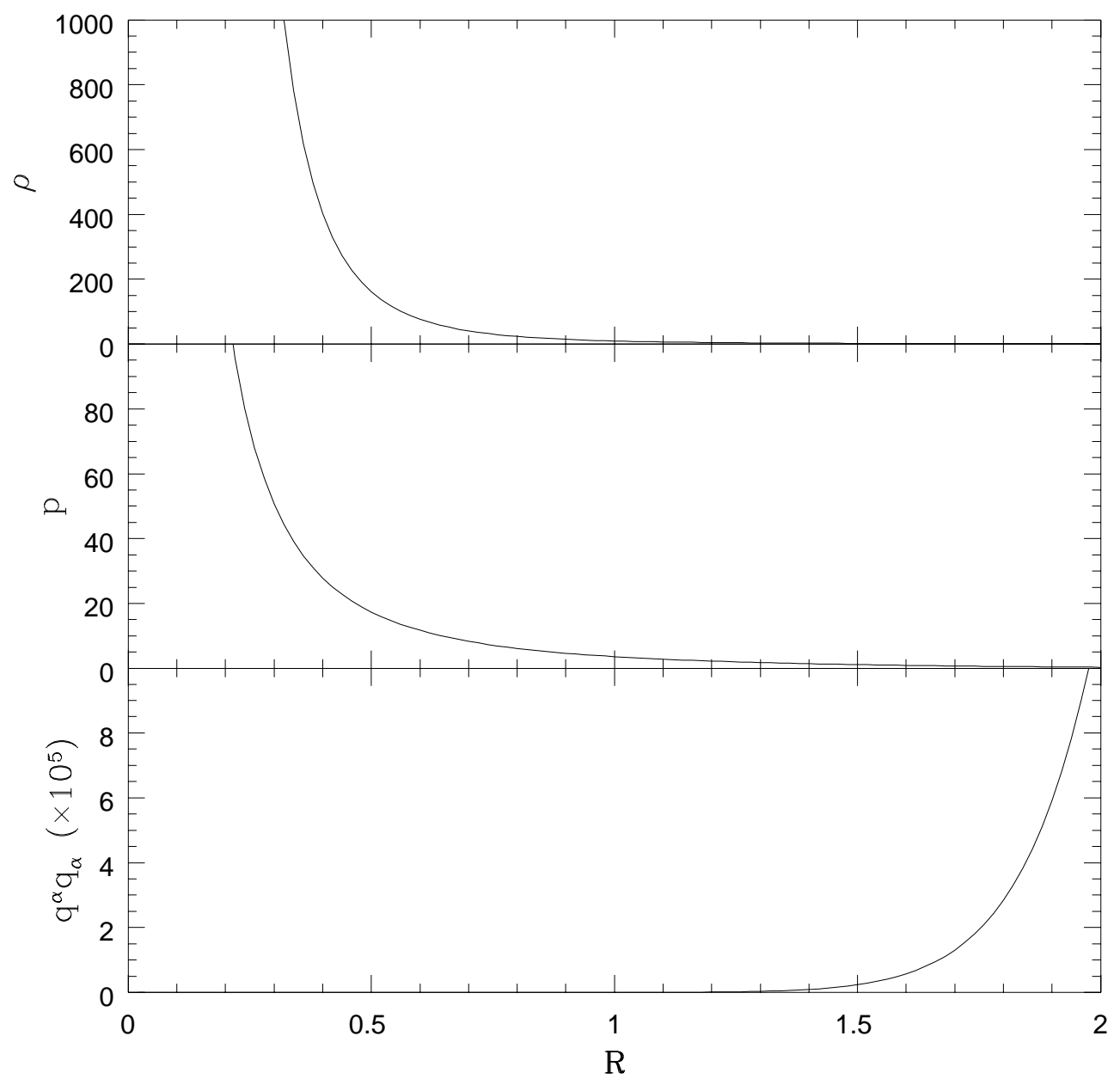

Figure 6: The "astrophysical" conditions $(c=1 / 15, t=-1)$. The geometrical radius $\mathbf{R}$ is in units of second, $\rho$ and $p$ are in units of $\sec ^{-2}$ and $q^{\alpha} q_{\alpha}$ is in units of $\sec ^{-4}$ 


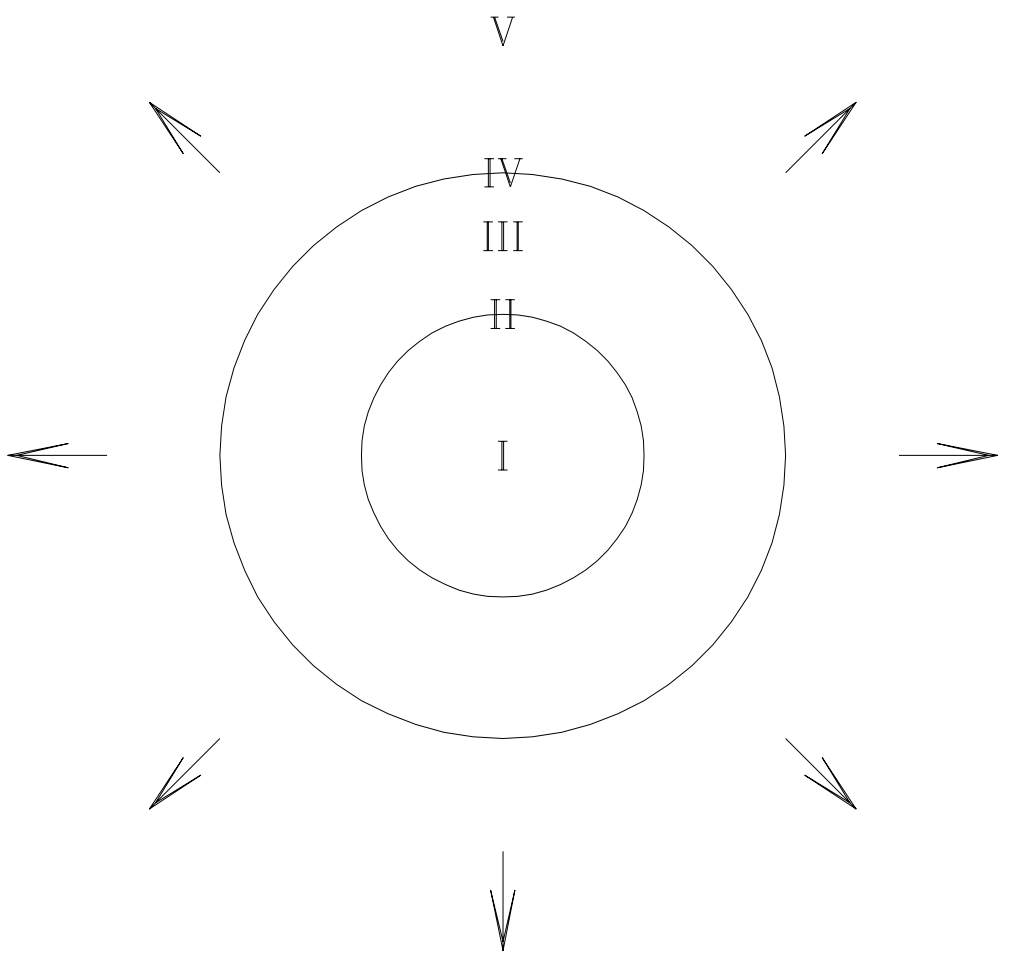

Figure 7: A scheme for the thick shell. The fluid with a heat flow fulfills the region III. The region I is the vacuum Minkowski spacetime inside the shell. Region $\mathrm{V}$ is the null radiation fluid (Vaidya's spacetime) outside the shell. The regions II and IV represent the $\Sigma_{1}$ and $\Sigma_{2}$ hypersurfaces, respectively. 
and

$$
R_{\Sigma_{1}}(V)=\beta(\tau)=A\left(r_{\Sigma 1}, t\right) r_{\Sigma_{1}} .
$$

The extrinsic curvature is given by

$$
K_{a b}=-n_{\alpha} \frac{\partial^{2} x^{\alpha}}{\partial \xi^{a} \partial \xi^{b}}-n_{\alpha} \Gamma_{\beta \gamma}^{\alpha} \frac{\partial x^{\beta}}{\partial \xi^{a}} \frac{\partial x^{\gamma}}{\partial \xi^{b}},
$$

where $n_{\alpha}$ is the unit normal vector, $x^{\alpha}$ refers to the equation of $\Sigma_{1}$ and $\xi^{a}$ take the values $\tau, \theta$ and $\phi$.

We can easily show that, using the Lichnerowicz [22] and O'Brien and Synge [23] junction conditions, it is impossible to match a spacetime with heat flow and a Minkowski spacetime. This can be due to the continuity of the radial flux of momentum across the hypersurface $\Sigma_{1}$ (see [24], page 283). Since we cannot make a smooth matching without a physical surface layer between them, we must introduce a thin shell with a energy-momentum tensor $T_{a b}$. Following Israel [25], the relation between the energy-momentum tensor of the layer and the extrinsic curvature (equation 54) of the spacetime is

$$
\left[K_{a b}^{I}-K_{a b}^{I I I}\right]_{\Sigma_{1}}=-\kappa\left(T_{a b}-g_{a b} T / 2\right),
$$

where $K_{a b}^{I}$ is the extrinsic curvature of the spacetime I and $K_{a b}^{I I I}$ is the extrinsic curvature of the spacetime III. The indices take the values $\tau, \theta$ and $\phi$, and

$$
T=g^{a b} T_{a b}
$$

The unit normal vectors of the spacetimes I and III can be written as

$$
n_{\alpha}^{I}=\left(-R_{\Sigma_{1}}^{*}, V^{*}, 0,0\right),
$$

and

$$
n_{\alpha}^{I I I}=\left(0, A\left(r_{\Sigma_{1}}, t\right), 0,0\right),
$$

where the symbol $*$ denotes differentiation with respect to the coordinate $\tau$.

Using the extrinsic curvature (equation 54) and the metrics (equations 48 and 49) and the equation $A^{2}(r, t)=t^{2} /\left(c t^{2}+r^{2}\right)$ we have

$$
\begin{gathered}
K_{\tau \tau}^{I}=\left[R^{*} V^{* *}-V^{*} R^{* *}\right]_{\Sigma_{1}}, \\
K_{\theta \theta}^{I}=\left[V^{*} R\right]_{\Sigma_{1}},
\end{gathered}
$$




$$
\begin{gathered}
K_{\phi \phi}^{I}=\left[V^{*} R \sin ^{2} \theta\right]_{\Sigma_{1}}, \\
K_{\tau \tau}^{I I I}=\left[\frac{2 r\left(t^{*}\right)^{2} t^{2}}{\left(r^{2}+c t^{2}\right)^{2}}\right]_{\Sigma_{1}}, \\
K_{\theta \theta}^{I I I}=\left[\frac{r t^{2}\left(c t^{2}-r^{2}\right)}{\left(r^{2}+c t^{2}\right)^{2}}\right]_{\Sigma_{1}},
\end{gathered}
$$

and

$$
K_{\phi \phi}^{I I I}=\left[\frac{r t^{2}\left(c t^{2}-r^{2}\right)}{\left(r^{2}+c t^{2}\right)^{2}} \sin ^{2} \theta\right]_{\Sigma_{1}} .
$$

Thus, substituting equations (59)-(64) into equation (55) we get

$$
\begin{gathered}
T_{\tau \tau}=\frac{1}{\kappa}\left[\frac{2 V^{*}}{R}-\frac{2\left(c t^{2}-r^{2}\right)}{r t^{2}}\right]_{\Sigma_{1}}, \\
T_{\theta \theta}=\frac{1}{\kappa}\left[R^{2}\left(R^{*} V^{* *}-V^{*} R^{* *}\right)-R V^{*}+\frac{r t^{2}\left(c t^{2}-3 r^{2}\right)}{\left(c t^{2}+r^{2}\right)}\right]_{\Sigma_{1}},
\end{gathered}
$$

and

$$
T_{\phi \phi}=T_{\theta \theta} \sin ^{2} \theta
$$

The energy-momentum tensor of a perfect fluid can be constructed as

$$
T_{a b}=\sigma u_{a} u_{b}+\eta\left(\Theta_{a} \Theta_{b}+\Phi_{a} \Phi_{b}\right),
$$

where

$$
\begin{gathered}
u_{a}=\delta_{a}^{\tau}, \\
\Theta_{a}=\beta \delta_{a}^{\theta},
\end{gathered}
$$

and

$$
\Phi_{a}=\beta \sin \theta \delta_{a}^{\phi} .
$$

Comparing equations (65)-(67) and (68), we can write that

$$
\kappa \sigma=\left[\frac{2 V^{*}}{R}-\frac{2\left(c t^{2}-r^{2}\right)}{r t^{2}}\right]_{\Sigma_{1}},
$$

and

$$
\kappa \eta=\left[\left(R^{*} V^{* *}-V^{*} R^{* *}\right)-\frac{V^{*}}{R}+\frac{c t^{2}-3 r^{2}}{r t^{2}}\right]_{\Sigma_{1}} .
$$


The equations (72)-(73) can be rewritten as

$$
\kappa \sigma=\left[\frac{2\left(1+R^{* 2}\right)^{\frac{1}{2}}}{R}-\frac{2\left(c t^{2}-r^{2}\right)}{r t^{2}}\right]_{\Sigma_{1}}
$$

and

$$
\kappa \eta=\left[-\frac{\left(1+R^{* 2}\right)^{\frac{1}{2}}}{R}-\frac{R^{* *}}{\left(1+R^{*^{2}}\right)^{\frac{1}{2}}}+\frac{c t^{2}-3 r^{2}}{r t^{2}}\right]_{\Sigma_{1}} .
$$

\subsection{Energy Conditions for the Thin Shell}

Differentiating the equation (53) with respect to $\tau$ we get

$$
R_{\Sigma_{1}}^{*}=\left[\frac{2 r^{3}}{t\left(c t^{2}+r^{2}\right)}\right]_{\Sigma_{1}}
$$

Thus, we can see that the hypersurface $\Sigma_{1}$ collapses since $t<0$ thus $R^{*}<0$.

The weak energy conditions are given by $\sigma \geq 0$ and $\sigma+\eta \geq 0$, thus from the first condition we get

$$
\left\{c t^{2}+r^{2}\left[1+\frac{4 r^{6}}{t^{2}\left(c t^{2}+r^{2}\right)^{2}}\right]^{\frac{1}{2}}\right\}_{\Sigma_{1}} \geq\left(c t^{2}-r^{2}\right)_{\Sigma_{1}}
$$

which is always satisfied.

¿From the second condition we have

$$
\left[\frac{R^{* *}}{\left(1+R^{* 2}\right)^{\frac{1}{2}}}\right]_{\Sigma_{1}} \leq\left[\frac{1+R^{* 2}}{R\left(1+R^{*^{2}}\right)^{\frac{1}{2}}}-\frac{1}{R}\right]_{\Sigma_{1}}=F_{1}
$$

In order to hold the dominant energy conditions we must have the conditions $\sigma \geq 0$ and $\sigma+\eta \geq 0$ and $\sigma-\eta \geq 0$. From this last condition we have

$$
\left[\frac{R^{* *}}{\left(1+R^{* 2}\right)^{\frac{1}{2}}}\right]_{\Sigma_{1}} \geq\left[-\frac{3\left(1+R^{* 2}\right)^{\frac{1}{2}}}{R}+\frac{3 c t^{2}-5 r^{2}}{r t^{2}}\right]_{\Sigma_{1}}=F_{2}
$$

In order to hold the strong energy conditions we must have the conditions $\sigma+\eta \geq 0$ and $\sigma+2 \eta \geq 0$. From this last condition we have 


$$
\left[\frac{R^{* *}}{\left(1+R^{*^{2}}\right)^{\frac{1}{2}}}\right]_{\Sigma_{1}} \leq\left[-\frac{2 r}{t^{2}}\right]_{\Sigma_{1}}=F_{3} .
$$

Thus, we can conclude that $R^{* *} \leq 0$. It is easy to show that $F_{1} \geq 0$ and that equation (78) is always satisfied.

Finally, we can show that $F_{2}<F_{3}$. Thus, in order to hold all the energy conditions we must have

$$
F_{2} \leq\left[\frac{R^{* *}}{\left(1+R^{* 2}\right)^{\frac{1}{2}}}\right]_{\Sigma_{1}} \leq F_{3} .
$$

The above equation implies in two conditions, that can be written in terms of the self-similar variable $z$, given by

$$
\begin{aligned}
& {\left[z^{8}+(6 c-4) z^{6}+\left(9 c^{2}-1\right) z^{4}-2 c z^{2}-c^{2}\right]_{\Sigma_{1}}<0(82)} \\
& {\left[-2 z^{6}+(6 c-1) z^{4}-2 z^{2}-1+\left(2 c-5 z^{2}\right) \sqrt{\left(c+z^{2}\right)^{2}+4 z^{6}}\right]_{\Sigma_{1}}<0(.83)}
\end{aligned}
$$

For the entire range of $z$ and $c$ imposed by the energy conditions of the thick shell, we can see that they are all satisfied. Thus, the Minkowski spacetime can be identified as the region above the $x_{2}(c)$ (the condition energy frontier), for example, in the figures (8) and (9).

\section{Matching Regions III and V}

In this section we present and analyze the junction conditions for regions III and V (Vaidya's spacetime [15]), as shown in the figure 7.

\subsection{Junction Conditions for the region IV}

The Vaidya's metric is given by

$$
d s_{V}^{2}=[1-2 m(\mathbf{v}) / \mathbf{r}] d \mathbf{v}^{2}-2 d \mathbf{r} d \mathbf{v}-\mathbf{r}^{2} d \theta^{2}-\mathbf{r}^{2} \sin ^{2} \theta d \phi^{2},
$$

where $m(\mathbf{v})$ is an arbitrary function the time $\mathbf{v}$.

The metric of the hypersurface $\Sigma_{2}$ is given by

$$
d s_{I V}^{2}=d v^{2}-\gamma^{2}(v)\left(d \theta^{2}+\sin ^{2} \theta d \phi^{2}\right) .
$$


¿From the junction condition

$$
\left(d s_{I I I}^{2}\right)_{\Sigma_{2}}=\left(d s_{I V}^{2}\right)_{\Sigma_{2}}=\left(d s_{V}^{2}\right)_{\Sigma_{2}},
$$

we obtain

$$
\begin{gathered}
\frac{d t}{d v}=A\left(t, r_{\Sigma_{2}}\right)^{-1}, \\
A\left(t, r_{\Sigma_{2}}\right) r_{\Sigma_{2}}=\gamma(v)=\mathbf{r}_{\Sigma_{2}}(\mathbf{v}),
\end{gathered}
$$

and

$$
\left(\frac{d \mathbf{v}}{d v}\right)_{\Sigma_{2}}^{-2}=\left(1-\frac{2 m}{\mathbf{r}}+2 \frac{d \mathbf{r}}{d \mathbf{v}}\right)_{\Sigma_{2}},
$$

where $v$ is a time coordinate defined only on $\Sigma_{2}$.

The unit normal vectors to $\Sigma_{2}$ (for details see [26]) are given by

$$
\begin{gathered}
n_{\alpha}^{I I I}=A\left(t, r_{\Sigma_{2}}\right) \delta_{\alpha}^{1} \\
n_{\alpha}^{V}=\left(1-\frac{2 m}{\mathbf{r}}+2 \frac{d \mathbf{r}}{d \mathbf{v}}\right)_{\Sigma_{2}}^{-1 / 2}\left(-\frac{d \mathbf{r}}{d \mathbf{v}} \delta_{\alpha}^{0}+\delta_{\alpha}^{1}\right)_{\Sigma_{2}} .
\end{gathered}
$$

The non-vanishing extrinsic curvature components are given by

$$
\begin{gathered}
K_{v v}^{I I I}=-\left[\left(\frac{d t}{d v}\right)^{2} A^{\prime}\right]_{\Sigma_{2}}, \\
K_{\theta \theta}^{I I I}=\left[r(A r)^{\prime}\right]_{\Sigma_{2}}, \\
K_{\phi \phi}^{I I I}=K_{\theta \theta}^{I I I} \sin ^{2} \theta, \\
K_{v v}^{V}=\left[\frac{d^{2} \mathbf{v}}{d v^{2}}\left(\frac{d \mathbf{v}}{d v}\right)^{-1}-\left(\frac{d \mathbf{v}}{d v}\right) \frac{m}{\mathbf{r}^{2}}\right]_{\Sigma_{2}}, \\
K_{\theta \theta}^{V}=\left[\left(\frac{d \mathbf{v}}{d v}\right)\left(1-\frac{2 m}{\mathbf{r}}\right) \mathbf{r}+\frac{d \mathbf{r}}{d v} \mathbf{r}\right]_{\Sigma_{2}}, \\
K_{\phi \phi}^{V}=K_{\theta \theta}^{V} \sin ^{2} \theta,
\end{gathered}
$$

where the prime denotes differentiation with respect to the coordinate $r$. ¿From the equations (93) and (96) we have 


$$
\left[\left(\frac{d \mathbf{v}}{d v}\right)\left(1-\frac{2 m}{\mathbf{r}}\right) \mathbf{r}+\frac{d \mathbf{r}}{d v} \mathbf{r}\right]_{\Sigma_{2}}=\left[r(A r)^{\prime}\right]_{\Sigma_{2}} .
$$

With the help of equations (87), (88), (89), we can write (98) as

$$
m=\left\{\frac{r A}{2}\left[1+\left(\frac{r \dot{A}}{A}\right)^{2}-\left(1+\frac{r A^{\prime}}{A}\right)^{2}\right]\right\}_{\Sigma_{2}},
$$

which is the total energy entrapped inside the hypersurface $\Sigma_{2}$ [27] and where the dot denotes differentiation with respect to the coordinate $t$.

Using equations (92) and (95) and following the same procedure described in [24], we can finally write

$$
(p)_{\Sigma_{2}}=(q A)_{\Sigma_{2}}
$$

Substituting the equations for $p$ and $q$ into (100) we get (see figures (8) and $(9))$

$$
2 z_{\Sigma_{2}}^{3}+(1+3 c) z_{\Sigma_{2}}^{2}+2 c z_{\Sigma_{2}}-2 c=0
$$

The hypersurface of the matching is always inside the interval allowed by the energy conditions of the thick shell.

\subsection{Inexistence of an Apparent Horizon}

Below we prove that the singularity at $t=0$ and $\mathbf{R}=0$ is naked for the thick shell.

Its is known that on the apparent horizon $g_{t t}$ changes its sign. We say that a black hole is formed during a collapse process when its boundary hypersurface cross inwards an apparent horizon. Since at the initial stage $g_{t t}$ is positive, the condition for the inexistence of an apparent horizon is

$$
\left(1-\frac{2 m}{\mathbf{R}}\right)_{\Sigma_{2}}>0
$$

Considering the matching with Vaidya's solution we can rewrite equation (102) as

$$
-\left[\frac{(1-z)\left(z^{2}+c\right) z}{\left(1-z^{2}\right)}\right]_{\Sigma_{2}}<\frac{1}{2}
$$




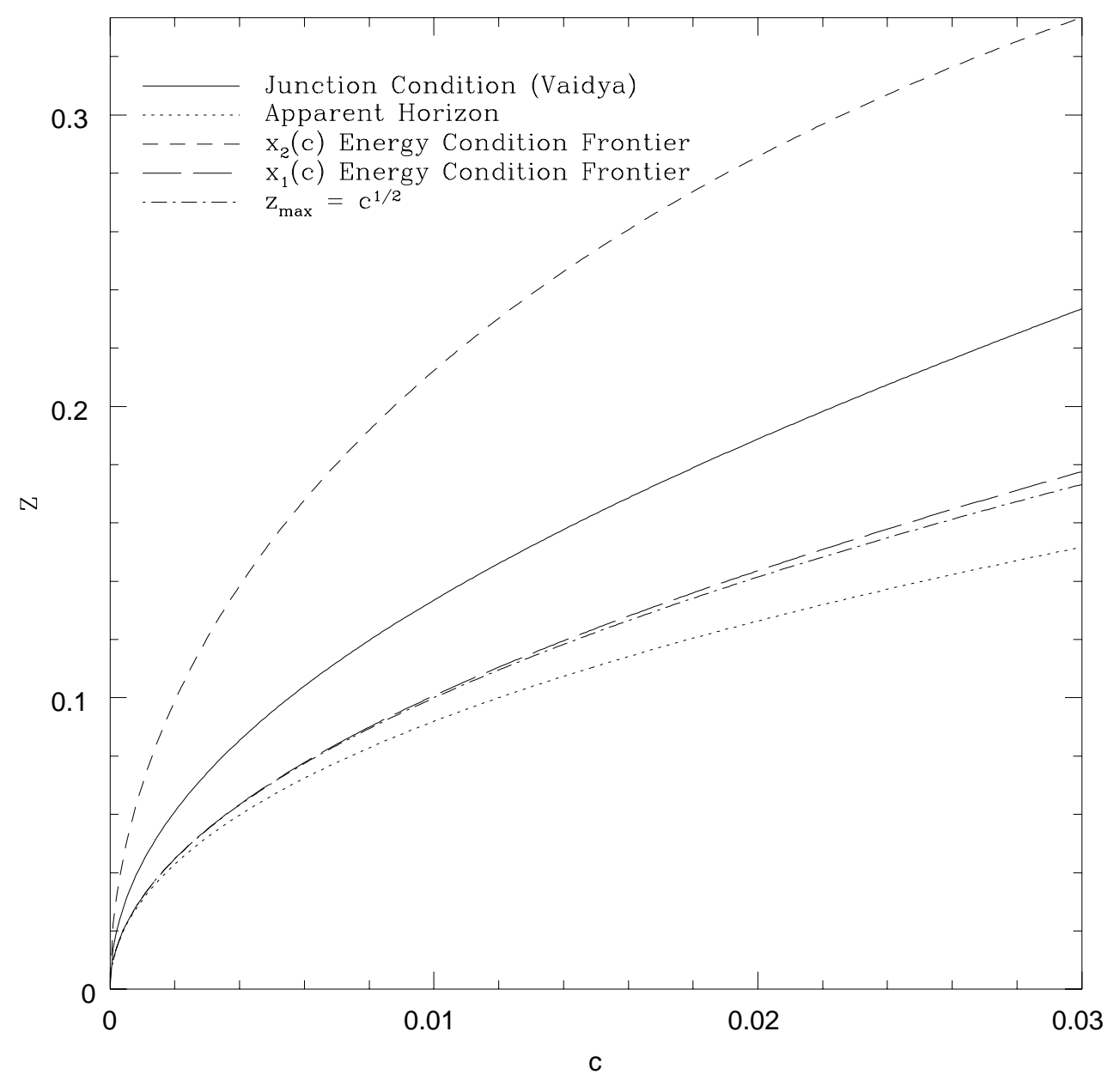

Figure 8: The energy condition frontiers, apparent horizon and junction condition, in a $c-z$ diagram. 


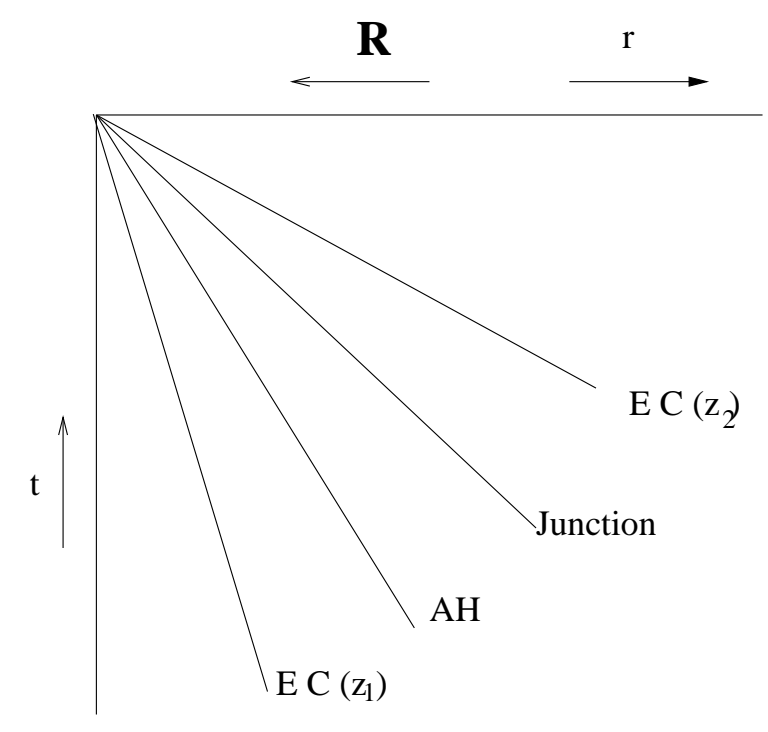

Figure 9: The energy condition frontiers, apparent horizon and junction condition, in the t- $\mathbf{R}$ diagram. The energy frontiers $z_{1}$ and $z_{2}$ correspond to $x_{1}$ and $x_{2}$, respectively. EC denotes energy conditions and $\mathrm{AH}$ denotes apparent horizon. 
Since the ranges of $c$ and $z_{\Sigma}$ are $0 \leq c \leq 1 / 15$ and $0 \leq z_{\Sigma}<1 / 3$, respectively, it is easy to verify that this inequality is always satisfied.

\section{Conclusions}

In this paper, we have studied a complete class of solutions, (shear-free, conformally flat, self-similar) which represents gravitational collapse of a fluid with heat flow. The fluid satisfies all the energy conditions only in the region $z_{1}<z<z_{2}$, where $z$ denotes the self-similar variable. Thus, to have a physically realistic model, one needs to do some kind of "surgery", that is, cutting the spacetime along the two hypersurfaces $z=z_{1,2}$ and then matching the part $z_{1}<z<z_{2}$ to some other regions of spacetime. Since these hypersurfaces are time-like, one can always be able to do so. Then, the resultant spacetime will represent gravitational collapse of a thick spherical shell. However, if we do not consider the strong energy condition, we can have a collapse of a full spherical body, instead of a thick shell. It has been shown that, at least for the self-similarity of first kind, the thick shell collapses always forming naked singularities.

This, together with the results obtained in $[7,8]$ show that the results obtained from the gravitational collapse of a perfect fluid [5] cannot be generalized to anisotropic fluid, and the formation of naked singularities depends not only on the shear of the fluid, but also on pressures and heat flow.

\section{Acknowledgments}

We would like very much to thank A. Z. Wang for valuable discussions and suggestions. The financial assistance from UERJ/FAPERJ (MFAdaS) and $\mathrm{CNPq}(\mathrm{JFVR})$ is gratefully acknowledged.

\section{References}

[1] R. Penrose, Riv. Nuovo Cimento, (Special Number) 1, 252 (1969).

[2] K.P. Thorne, in Magic Without Magic: John Archibald Wheeler, edited by J. Klauder (Friedmann, San Francisco, 1972), p.231. 
[3] For recent reviews, see, e.g., R. Penrose, in Black Holes and Relativistic Stars, edited by R.M. Wald (University of Chicago Press, 1998); A. Krolak, Prog. Theor. Phys. Suppl. 136, 45 (1999); P.S. Joshi, Pramana, 55, 529 (2000), P.S. Joshi, "Cosmic Censorship: A Current Perspective," gr-qc/0206087 (2002).

[4] K.-I. Nakao, K. Nakamura, and T. Mishima, "Hoop Conjecture and Cosmic Censorship in the Brane-World," gr-qc/0112067 (2001); D. Ida and K.-I. Nakao, "Isoperimetric inequality for higher-dimensional black holes," gr-qc/0204082 (2002).

[5] P.S. Joshi, N. Dadhich, and R. Maartens, Phys. Rev. D65, 101501 (2002).

[6] A. K. Raychaudhuri, Phys. Rev. 98, 1123, (1955).

[7] D. Schaefer, H. Goenner, Gen. Relativ. Grav. 32, 2119, (2000).

[8] A. Banerjee, S. Chaterjee and N. Dadhich "Spherical Collapse with Heat Flow and whitout horizon, (gr-qc 0209035) (2002).

[9] A.Z. Wang and H. P. de Oliveira, Phys. Rev. D56, 753 (1997); A.Z. Wang, J.F. Villas da Rocha, and N.O. Santos, Phys. Rev. D56, 7692 (1997); E.W. Hirschmann and A.Z. Wang, Phys. Lett. A249, 383 (1998); B. J. Carr and A. A. Coley, Class. Quantum Grav. 16, R31 (1999); A.Z. Wang and Y. Wu, Gen. Relativ. Grav. 31, 107 (1999); J.F. Villas da Rocha, A.Z. Wang and N.O. Santos, Phys. Lett. A255, 213 (1999); J.F. Villas da Rocha and A.Z. Wang, Class. Quantum Grav. 17, 2589 (2000); T. Harada and H. Maeda, Phys. Rev. D63, 084022 (2001); H.-C. Kim, S.-H. Moon, and J. H. Yee, JHEP, 0202, 046 (2002); S. M. Wagh and K. S. Govinder, "Spherically Symmetric, Self-Similar Spacetimes," gr-qc/0112035 (2001); H. Maeda, T. Harada, H. Iguchi, and N. Okuyama, "No Go Theorem for Kinematic Self-Similarity with A Polytropic Equation of State," gr-qc/0204039 (2002), and references therein.

[10] S. M. Wagh, R. V. Saraykar, P. S. Muktibodh, and K. S. Govinder, "Spherical Gravitational Collapse with Heat Flux and Cosmic Censorship," gr-qc/0112034 (2001), and references therein. 
[11] M.M. Som and N.O. Santos, J. Phys. A, 13, 191 (1981).

[12] B. Carter and R.N. Henriksen, Ann. Physique Supp. 14, 47 (1989).

[13] S.W. Hawking and G.F.R. Ellis, The Large Scale Structure of Spacetime, (Cambridge University Press, Cambridge, 1973).

[14] C. A. Kollassis, N. O. Santos, and D. Tsoubelis, Class. Quantum Grav. 5 (1988) 1329.

[15] P. C. Vaidya, Nature 171, 260, (1953)

[16] G.E. Barenblatt and Ya.B. Zel'dovich, Ann. Rev. Fluid. Mech. 4, 285 (1972).

[17] A.H. Cahill and M.E. Taub, Commu. Math. Phys. 21, 1 (1971).

[18] A. A. Coley, Class. Quantum Grav. 14, 87 (1997).

[19] C. F. Brandt, L.-M. Lin, J. F. Villas da Rocha and A.Z. Wang, Inter. J. Mod. Phys. D11, 155 (2002).

[20] E. Poisson, W. Israel, Phys. Rev D41, 1796 (1990).

[21] P.R.C.T. Pereira and A.Z. Wang, Gen. Relativ. Grav. 32, 2189 (2000); M. F. A. da Silva, L. Herrera, N. O. Santos, and A. Z. Wang, Class. Quantum Grav. 19, 3809 (2002).

[22] A. Lichnerowicz, Théories Relativistes de la Gravitation et de l'Eletromagnétisme (Paris: Masson) (1955).

[23] S. O’Brien, and J. L. Synge, Comm. Dublin Inst. Adv. Stud., A No. 9, p. 1 (1952).

[24] W. B. Bonnor, A. K. G. de Oliveira and N. O. Santos, Physics Report 181, 269 (1989).

[25] W. Israel, Nuovo Cimento 44B, 4349 (1966).

[26] N. O. Santos, Month Not. Roy. Astr. Soc. 216, 403 (1985).

[27] M. E. Cahill, G. C. McVittie, J. Math Phys. 11, 1382, (1970). 